\title{
Will alternative immediate care services reduce demands for non-urgent treatment at accident and emergency?
}

P Coleman, R Irons, J Nicholl

\begin{abstract}
Objectives-To estimate the potential of general practice, minor injury units, walk in centres and NHS Direct to reduce nonurgent demands on accident and emergency $(A \& E)$ departments taking into account the patient's reasons for attending A\&E.
\end{abstract}

Methods-A questionnaire survey and notes review of 267 adults presenting to the $A \& E$ department of a large teaching hospital in Sheffield, England, triaged to the two lowest priority treatment streams, was conducted over seven weeks. Using defined criteria, patients were classified by the suitability of the presenting health problem to be managed by alternative immediate care services or only by $A \& E$, and also by the likelihood, in similar circumstances, of patients presenting to other services given their reasons for seeking A\&E care.

Results-Full data were obtained for $96 \%$ of participants (255 of 267). Using objective criteria, it is estimated that $55 \%(95 \%$ CI $50 \%, 62 \%$ ) of the health problems presented by a non-urgent population attending A\&E are suitable for treatment in either general practice, or a minor injury unit, or a walk in centre or by self care after advice from NHS Direct. However, in almost one quarter (24\%) of low priority patients who self referred, $A \& E$ was not the first contact with the health services for the presenting health problem. The reason for attending $A \& E$ cited most frequently by the patients was a belief that radiography was necessary. The reason given least often was seeking advice from a nurse practitioner. Taking into account the objective suitability of the health problem to be treated elsewhere, and the reasons for attending $A \& E$ given by the patients, it is estimated that, with similar health problems, as few as $7 \%$ (95\% CI 3\%, 10\%) of the non-urgent A\&E population may be expected to present to providers other than $A \& E$ in the future.

Conclusions-The increasing availability of alternative services offering first contact care for non-urgent health problems, is likely to have little impact on the demand for $A \& E$ services.

(Emerg Med f 2001;18:482-487)

Keywords: immediate care services; NHS Direct
Against the background of increasing demands on accident and emergency (A\&E) departments, ${ }^{1}$ the debate on how to deliver health care appropriately to attenders who are neither accidents nor emergencies has focused on initiatives to encourage patients to present to their general practitioner (GP) ${ }^{2}$ or employing GPs in A\&E departments to manage the primary care component effectively and at more or less cost than hospital doctors. ${ }^{3-7}$ Recently, the debate has widened to include service models involving extended nursing roles such as minor injury units, ${ }^{8}$ nurse practitioners, ${ }^{9}$ walk in centres, ${ }^{10}$ and the telephone health advice line NHS Direct. ${ }^{11}$

We studied a sample of non-urgent $A \& E$ attenders to improve understanding of why patients present to $\mathrm{A} \& \mathrm{E}$ despite the availability of alternative services. We provide estimates of the potential that other models of health care have to alleviate demands for $A \& E$ services taking into account both the suitability of the health problem for treatment elsewhere, and the patients' reasons for presenting to $\mathrm{A} \& \mathrm{E}$.

\section{Methods}

SAMPLING

The study was undertaken between October and December 1997 in the A\&E department of a major teaching hospital in Sheffield, England. The A\&E department is an adult facility, receiving approximately 80000 new attenders each year. It is typical of other medium sized A\&E departments located in cities in England. On arrival, all new attenders are prioritised for treatment to colour coded triage streams. This A\&E operates a five colour system (black, red, blue, green, and yellow). The two indicating the lowest priority for treatment are "green", classified broadly as "new illnesses or injuries which are non-urgent", and "yellow", "injuries or long-standing problems where advice could have been sought elsewhere". In the study period 8057 adult attenders were assigned to either the "green" or the "yellow" treatment streams. Together these two low priority for treatment groups accounted for $61.5 \%$ of the all new $A \& E$ attenders, and formed the sample frame for the survey.

A sample size of 150 from each of the "yellow" and the "green" treatment streams was estimated to be sufficient to enable the percentage of patients in each stream who were treatable by services other than A\&E to be calculated within $+/-5 \%$ assuming that the true proportion is $10 \%$. Using a simple Latin square design $(7 \times 7 \times 7)$, the $\mathrm{A} \& \mathrm{E}$ nurse
Accepted for publication 18 December 2000 
recruited the first three patients assigned to each of the "yellow" and the "green" treatment streams in each of seven pre-selected "sessions". The start times of the sessions rotated daily through 24 hours for seven consecutive weeks. The design yielded a target sample of 147 patients from each of the "yellow" and "green" streams, totalling 294. The inclusion criteria were that participants should not be incapable through alcohol or drugs to complete a questionnaire, or have been referred to A\&E formally by letter from their general practitioner.

\section{DATA COLLECTION}

Data for the study were collected by a questionnaire completed by the patient shortly after arriving in $\mathrm{A} \& \mathrm{E}$. The $\mathrm{A} \& \mathrm{E}$ notes were then reviewed by a member of the research team. Ethical approval for the study was obtained from the local ethics committee, and signed consent to access the notes was given by each patient.

Patient perceptions of their health problem, how, where and when it occurred, and sociodemographic indicators were collected on the questionnaire. The most frequently quoted reasons for attending $\mathrm{A} \& \mathrm{E}$ published in the literature ${ }^{12-14}$ were configured into 30 statements (table 1). Participants were asked to indicate from the reasons listed all those that had been instrumental in their decision to attend $\mathrm{A} \& \mathrm{E}$ and also to add any "other"

Table 1 Reasons relevant to the patient's decision to attend $A \mathcal{E} E$

\begin{tabular}{|c|c|c|c|}
\hline \multirow[b]{2}{*}{ Reasons } & \multicolumn{2}{|c|}{ Frequency reported } & \multirow{2}{*}{$\begin{array}{l}\text { Strength of reason } \\
\text { for attending } A \mathcal{E} E\end{array}$} \\
\hline & Number & $\% *$ & \\
\hline \multicolumn{4}{|l|}{ Availability of other services } \\
\hline I don't have a GP & 6 & 3 & Medium \\
\hline My GP was not available & 12 & 5 & Medium \\
\hline $\begin{array}{l}\text { Nowhere else has } 24 \text { hour open access } \\
\text { Awareness of other services }\end{array}$ & \\
\hline I didn't know where else to go & 33 & 15 & Weak \\
\hline I am not aware of any other services & 33 & 16 & Weak \\
\hline I don't know what other services are open... & 47 & 23 & Weak \\
\hline I don't know if my GP is available & 16 & 8 & Weak \\
\hline \multicolumn{4}{|l|}{ Patient preferences } \\
\hline I didn't want to see my GP & 13 & 3 & Medium \\
\hline I can't always see the GP I would like & 6 & 2 & Medium \\
\hline I didn't want to bother my GP & 10 & 4 & Medium \\
\hline I wanted to see the nurse practitioner & 3 & 2 & Medium \\
\hline \multicolumn{4}{|l|}{ Positive experiences of $A \mathcal{E} \cdot E$} \\
\hline I've used A\&E before and was happy... & 84 & 38 & Strong \\
\hline I'm confident in the A\&E system & 74 & 32 & Strong \\
\hline \multicolumn{4}{|l|}{ Processes and patient's time } \\
\hline My GP would only refer me here anyway & 49 & 16 & Medium \\
\hline I would have to wait for a GP appointment & 62 & 27 & Medium \\
\hline I think I will be seen quicker here... & 32 & 13 & Medium \\
\hline \multicolumn{4}{|l|}{ Convenience of access } \\
\hline $\mathrm{A} \& \mathrm{E}$ is nearer than any other service & 29 & 11 & Medium \\
\hline It's easier to get to than any other service & 30 & 13 & Medium \\
\hline \multicolumn{4}{|l|}{ Perceptions of seriousness } \\
\hline I wanted to see a specialist & 17 & 4 & Strong \\
\hline I consider the condition an emergency & 24 & 11 & Strong \\
\hline I wanted to see a doctor asap & 58 & 24 & Strong \\
\hline I thought I might need to go into hospital & 14 & 4 & Strong \\
\hline \multirow{2}{*}{\multicolumn{4}{|c|}{ Seeking reassurance }} \\
\hline & & & \\
\hline I need reassuring that ...is not serious & 92 & 33 & Medium \\
\hline I wanted a second opinion & 23 & 5 & Medium \\
\hline \multicolumn{4}{|l|}{ Other directed } \\
\hline ...advised by friends/family & 47 & 20 & Medium \\
\hline ....advised by others & 61 & 23 & Strong \\
\hline \multicolumn{4}{|l|}{ Seeking particular services } \\
\hline I thought I needed a radiograph & 112 & 48 & Strong \\
\hline I thought I might need a tetanus injection & 14 & 7 & Medium \\
\hline I thought I might need a blood test & 8 & 1 & Medium \\
\hline I thought it needed stitches & 20 & 12 & Medium \\
\hline
\end{tabular}

^Estimated proportion (\%) of all non-urgent A\&E attenders giving this reason. reasons. Information about referral, diagnoses, processes of care and disposal was collected from the A\&E notes. The diagnoses in the notes were coded to the International Classification of Diseases 9th revision (ICD9). ${ }^{15}$ All the health problems within the range ICD9 800-999 (injury and poisoning) [ibid:735] were recoded as "trauma" and all else was recoded as "non-trauma".

\section{ANALYSIS}

The data were coded and analysed using SPSS for Windows. Population estimates of the total attendance at $\mathrm{A} \& \mathrm{E}$ with less urgent conditions were calculated after weighting the numbers in the sample recruited from the "green" and "yellow" streams to reflect their proportions in the sample frame (92\% "green" and $8 \%$ "yellow") and 95\% confidence intervals were calculated for key estimates. To enable the effect of the weighting to be assessed, unless otherwise stated, all the numbers reproduced in this paper are raw data and all the population estimates are based on weighted numbers.

\section{SUITABILITY FOR TREATMENT BY OTHER} PROVIDERS

The patients were classified into subgroups based on their suitability for treatment in other settings (box 1). The alternative models of healthcare considered were general practice, a nurse led minor injury unit (MIU), a walk in centre, with the capacity to treat both minor injuries and minor medical conditions, and the national telephone advice line NHS Direct that can advise on the management of minor health problems not needing medical care.

The criteria we used to identify patients suitable for treatment in general practice were developed together with GPs in a UK study. ${ }^{16}$ The criteria to identify suitability for treatment in the MIU were developed by applying the nurse practitioner protocols in use at the MIU located at another hospital site in Sheffield to both the description of the health problem given by the patient on the questionnaire, and the processes of care received as recorded in the notes. Walk in centres will have various specification ${ }^{10}$ but the model we assumed to distinguish it from the hospital-based MIU was on a site remote from an $\mathrm{A} \& \mathrm{E}$ department (for example in a railway station or high street setting) staffed by nurses working within protocols similar to those used in the MIU that permit some treatments, advice, and prescribing to be carried out, but that unlike the MIU do not have direct access to radiography facilities. The group identified as appropriate for NHS Direct were those whose processes of care defined them as suitable to receive advice to "self care" (box 1). The group identified as suitable for treatment in a traditional A\&E setting was the "default" category so defined if none of the criteria for suitability for treatment or advice in the other models of care were met. 


\section{Box 1 Criteria for identifying suitability for treatment by other services in a sample of attenders at $A \& E$ with non-urgent conditions}

(1) Suitable for treatment in general practice

- Self referred

- Registered with a GP

- Not an accident except at home

- No treatment other than a prescription, or a dressing, sling, bandage, or steristrip, or advice

- No investigations

- Discharged home or to GP

(2) Suitable for treatment in a minor injury unit

- Self referred

- Comparison of the description of the health problem given by the patient shortly after their arrival in $\mathrm{A} \& \mathrm{E}$, and the nurse practitioner protocols in use at the minor injury unit (MIU) on a different trust site in the city

- Not referred to other hospital services on the day of attendance

- Availability of the MIU service (0800 2000 hours $\times 7$ days/week) at time of registration

(3) Suitable for treatment in a walk in centre

- Self referred

- No treatment other than a prescription in line with nurse practitioner protocols, or a dressing, sling, bandage, or steri-strip, or advice

- No hospital-based investigation services (for example, radiography, computed tomography, etc)

- Not referred to other hospital services on the day of attendance

- Availability of the walk in service (07002200 hours $\times 7$ days/week) at time of registration

(4) Suitable for advice from NHS Direct to self care

- Self referred

- Access to a telephone at home if the health problem occurred in the home

- No investigations or prescription of medicine

- No treatment other than advice

- No known barriers (language or health problems) prohibiting use of the telephone

- Discharged home

(5) Suitable for treatment in an A\&E setting

- "default" of not meeting the criteria for inclusion elsewhere

REASONS FOR THE PATIENT'S DECISION TO

ATTEND A\&E

We classified the reasons for attending $A \& E$ given by the patients on the questionnaire into "strong", "medium" and "weak" (table 1). "Strong" reasons for attending A\&E were those we considered to have low potential for changing consulting behaviour. Reasons scored as "medium" were those which offered moderate potential for changing the decision to attend A\&E. The reasons graded as "weak" were those we considered to offer the highest potential for changing the decision to attend A\&E.

The reasons, "seeking a radiograph", (seeking particular A\&E services) or "I consider the condition as emergency" (perceptions of seriousness), were classified as "strong" reasons. Reasons reflecting positive experiences of $A \& E$ in the past were also graded as "strong" in that they reflected previous patterns of consulting behaviour that might be difficult to influence. The reason being "...advised to attend by someone else" was classified as "medium" if the source of the advice was "friends or family", and "strong" if the advice came from "others" in a formal relationship with the patient, such as a works nurse. Reasons reflecting "convenience of A\&E services", and "seeking reassurance" or "not wishing to contact the GP" were graded "medium" on the grounds that other services may become more convenient, and services other than A\&E may provide reassurance. Not being registered or wishing to contact a GP does not in itself preclude the future possibility of consulting providers other than A\&E or a GP, so these reasons were also classified as "medium". Reasons grouped under "lack of awareness of other services" were graded "weak" assuming that changes in consulting behaviour may be influenced by effective publicity (table 1). Eight reasons given by patients in the "other" category (box 2) were not weighted and therefore not graded formally or included in the analysis.

Box 2 "Other" reasons given for attending $A \& E$

- If not busy I thought $\mathrm{A} \& \mathrm{E}$ would prescribe some antibiotics

- Emergency dentist failed to inspire confidence

- A\&E obvious place for this accident

- After three days on antibiotics no improvement

- Not satisfied with GP seen

- Confirmation of injury to support claim for compensation

- I thought it may be bruised, sprained or chipped

- Treatment all under one roof including specialist treatment

The pattern of graded reasons given by each patient was identified. Those combinations containing any "strong" reason for attending A\&E with low potential to change, were taken as an indication that in similar circumstances and health problems in the future the patient would present to $\mathrm{A} \& \mathrm{E}$ irrespective of the other reasons or services.

\section{Results}

RESPONSE

Two hundred and sixty seven attenders agreed to complete a questionnaire. Of these, two questionnaires were lost and one was handed 


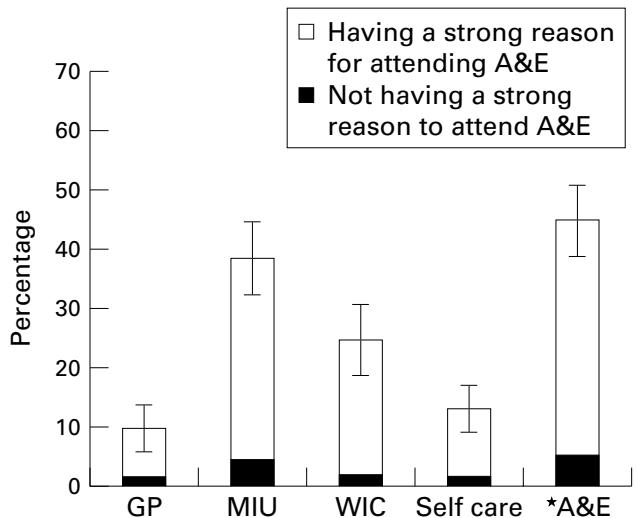

Figure 1 Estimated proportions (and 95\% confidence intervals shown by bars) of a non-urgent population of attenders at $A \mathcal{E} E$ who were suitable for treatment in other settings. ${ }^{\star}$ Only exclusive group.

in uncompleted. Twelve of those who completed a questionnaire did not sign the consent to access the notes. Full data (a completed questionnaire and notes review) were obtained for $96 \%$ of the forms distributed (255 of 267). The achieved sample consisted of 119 from attenders triaged to the "yellow" stream and 136 from the "green" stream. The age-sex distribution of the unweighted sample was $62 \%$ men $38 \%$ women, and $57 \%$ were aged under 35 years.

ESTIMATED CHARACTERISTICS OF ATTENDANCE AT A\&E IN A NON-URGENT POPULATION

After weighting of the achieved sample the estimated ratio of men to women in the population was $2: 1(68 \% \mathrm{M} ; 32 \% \mathrm{~W})$. The mean age was 34 years. It is estimated that $81 \%$ were self referred, the remaining $19 \%$ having been advised to attend by someone in authority to them such as a manager, or health related personnel. The recoding of the health problems yielded an overall case mix of $80 \%$ trauma: $20 \%$ non-trauma. Slightly more than one quarter $(27 \%)$ of the health problems occurred at home but almost two thirds of the study population (63\%) travelled to $\mathrm{A} \& \mathrm{E}$ from home. In self referred patients, $24 \%$ of those delaying more than six hours had consulted (or tried to consult) another health professional for the same problem before attending $\mathrm{A} \& \mathrm{E}$.

Table 2 Comparison of characteristics of a non-urgent population of $A \mathcal{E} E$ attenders not suitable for treatment in other settings and Others (proportions based on weighted numbers)

\begin{tabular}{llll}
\hline Characteristics & $\begin{array}{l}A \mathcal{E} E \\
(n=103) \%\end{array}$ & $\begin{array}{l}\text { Others } \\
(n=152) \%\end{array}$ & $p$ Value \\
\hline $\begin{array}{l}\text { Aged under 35 years } \\
\text { Sex: male }\end{array}$ & 66 & 53 & $<0.05$ \\
Occurred & 69 & 67 & $\mathrm{NS}$ \\
Home & 26 & & \\
Work & 45 & 29 & \\
Other & 29 & 48 & $<0.001$ \\
Started journey to A\&E from home & 55 & 70 & $<0.01$ \\
Delay >6 hours before presenting to A\&E (n=127) \\
(delay >6 hours) Previous contact with health & 35 & 63 & $<0.0001$ \\
$\quad$ professionals & 45 & 20 & $<0.01$ \\
In paid employment (n=184) & 76 & 69 & $\mathrm{NS}$ \\
Manual occupation & 57 & 43 & $\mathrm{NS}$ \\
Arrived by private car & 71 & 78 & $\mathrm{NS}$ \\
Health status: excellent/good & 80 & 92 & $<0.01$ \\
\hline
\end{tabular}

SUITABILITY OF THE NON-URGENT POPULATION FOR TREATMENT BY OTHER PROVIDERS

We calculated that two thirds of patients in the "yellow" stream and $54 \%$ in the "green" stream, were suitable for treatment elsewhere, yielding an overall estimate of 55\% (95\% CI $50 \%, 62 \%$ ) who could be treated in at least one of the other services considered. An estimated $13 \%$ might have been directed away from $A \& E$ to selfcare by NHS Direct ( $95 \%$ CI $9 \%, 17 \%)$, and $25 \%$ by the walk in centre $(95 \%$ CI $19 \%$, $31 \%$ ) (fig 1). General practice was suitable for $10 \%(95 \%$ CI $6 \%, 14 \%)$, and the MIU could have treated 38\% (95\% CI 32\%, 44\%). With the exception of those suitable for treatment only in $\mathrm{A} \& \mathrm{E}$, the numbers suitable for treatment by other providers (fig 1) are not exclusive. The largest overlap in suitable service provision was between walk in centres and NHS Direct, where 28 (sample numbers) satisfied the criteria for inclusion in both (an estimated $11 \%$ of the population after weighting).

ESTIMATED DIFFERENCES BETWEEN THE POPULATION SUITABLE FOR TREATMENT ONLY IN A\&E AND OTHERS

The estimated age profile of those suitable for treatment only in $\mathrm{A} \& \mathrm{E}$ is younger than all the others. A higher proportion in the $\mathrm{A} \& \mathrm{E}$ group was aged under 35 years (table 2). No differences were observed between those suitable only for A\&E and all the others, in the proportions that arrived at $\mathrm{A} \& \mathrm{E}$ by a private car, but 14 of the 18 in our sample who arrived by taxi (unweighted numbers), were in the A\&E group. Of the 21 in the sample who had home addresses outside the Sheffield boundary (an estimated $9 \%$ of the population after weighting), the health problems of 13 were suitable for treatment only in A\&E. Looking solely at those who self refer, the only population-based differences in the proportions of attenders at $\mathrm{A} \& \mathrm{E}$ assigned to low priority treatment streams between those suitable for treatment only in A\&E and the others which retained statistical significance was in delaying less than six hours before presenting to $\mathrm{A} \& \mathrm{E}$ (table 2).

REASONS FOR ATTENDING A\&E

The reasons reported most frequently for attending $A \& E$ in our sample were: "I thought I needed a radiograph .." $(n=112)$, and being "...advised to come to A\&E by someone else" $(\mathrm{n}=108)$ (table 1). The sources of advice in this second group divided between informal "friends or family" and "others". The reason reported least often was "seeking advice from a nurse practitioner".

In the pattern of reasons for attending $A \& E$ provided by each patient, 34 of the 255 (unweighted numbers) did not include at least one "strong" reason with a low potential to change their decision to attend A\&E. In the $55 \%$ of the population who were suitable for treatment by other providers $12 \%$ (95\% CI $6 \%, 18 \%$ ) did not include at least one strong reason for attending A\&E. Thus the estimated proportion of this non-urgent $A \& E$ population 
who might appropriately (defined according to various objective measures) and realistically (as defined by patient perceptions of need) divert to immediate care providers other than $A \& E$ in similar circumstances in the future, was just 20 of 255 (estimated $7 \%$ after weighting (95\% CI $3 \%, 10 \%))$.

\section{Discussion}

PRINCIPAL FINDINGS

Objective measures indicate that newly developing, alternative immediate care services have the potential to treat $55 \%$ (95\% CI 50\%, 62\%) of patients currently attending A\&E with less urgent conditions. However, the patterns of the patients' reasons for presenting to $\mathrm{A} \& \mathrm{E}$ observed in our study, suggest that the real extent to which these services will deflect demands for non-urgent care away from $A \& E$ is much less. The belief that a radiograph is necessary is clearly an important factor in the decision to present to $\mathrm{A} \& \mathrm{E}$, as are disparities between the professional view and the patient's perceptions of the seriousness of the health problem, and expectations of care. Previous patterns of consulting behaviour and experiences of services both elsewhere and in $\mathrm{A} \& \mathrm{E}$ are also important factors. In our study just $12 \%$ of those with health problems defined objectively as suitable for treatment elsewhere, did not include a "strong" reason for presenting to A\&E. It is estimated therefore that realistically, the alternative service models we considered will absorb just $7 \%(95 \%$ CI $3 \%, 10 \%)$ of the patient population currently presenting to A\&E with non-urgent health problems.

VALIDITY/COMPARABILITY WITH OTHER STUDIES There is no reason to believe that our sample from two low priority treatment groups in a typical A\&E department is not representative of patients in the same treatment categories seen in other A\&E departments, or to doubt the wider generalisability of the results. The proportion of all A\&E attenders who could be treated in primary care or minor injury setting has been estimated previously as $10 \%-40 \%{ }^{17}$ and $40 \%-50 \%{ }^{18}$ respectively. These proportions are consistent with our findings of $55 \%$ of the non-urgent caseload that could be treated in another setting. Our finding of $24 \%$ of self referred attenders who have consulted a health professional previously for the same health problem before presenting to $\mathrm{A} \& \mathrm{E}$ is similar to the proportion of $21 \%$ reported in an earlier study. ${ }^{13}$

MECHANISMS AND IMPLICATIONS FOR POLICYMAKERS

The recent changes in primary care ${ }^{19}$ may require that the criteria we used to identify the subgroup of patients suitable for treatment in general practice be reviewed. It is nevertheless remarkable that using a validated algorithm, ${ }^{16}$ general practice was identified as suitable for managing only an estimated $10 \%(95 \%$ CI $6 \%$, $14 \%$ ) of the study population and was revealed as a relatively unimportant alternative to $\mathrm{A} \& \mathrm{E}$ for non-urgent health problems. In contrast, based on current provision in Sheffield, up to
$38 \%$ of the non-urgent caseload in A\&E could be treated suitably in a MIU. If the availability of the MIU was extended to 24 hours $\times 7$ days it might be able to absorb $72 \%$ (95\% CI $66 \%$, $78 \%$ ) of the non-urgent A\&E caseload. We did not estimate what the costs of such changes would be but the methods used in the study would permit such comparisons to be made.

Most of the difference between the service potential of an MIU and a walk in centre to relieve the non-urgent demand for $\mathrm{A} \& \mathrm{E}$ is explained by access to radiography facilities. Radiography was not included in the specifications for the first 19 walk in centres declared ${ }^{10}$ but radiographs are available within strict guidelines in the model of MIU we considered. In our study, 58 radiographs were carried out in those believing a radiograph was required, and a further 37 in those not expecting to have one, indicating considerable disparity between patient expectations and professional views of treatment required. Whether or not all the radiographs ordered were clinically necessary was not answerable within our design, but "I thought I needed a radiograph .." was the reason cited most often by our sample (table 1). The availability of radiographic services is clearly an important factor, and is likely to have an impact on the utilisation of services that are remote from radiographic facilities. On the other hand, only three patients indicated specifically that they wished to see a nurse practitioner. This again raises issues around patient expectations and the use of nurse-led services by those seeking advice from a doctor. These considerations are of great importance in attempts to encourage patients to choose appropriate services such as in the department of health's recent campaign "Choosing the right remedy" ${ }^{20}$ These campaigns are likely to falter on a confusion of clinical needs and patient perceptions and beliefs. Advice to go to A\&E or MIU "if you need urgent hospital treatment" is predicated on mistaken assumptions about the capacity of patients and their friends and family to assess this accurately.

STRENGTHS AND WEAKNESSES OF THE STUDY Our confidence in the study is based on its design and rigorous execution of the methods that permitted patients' perceptions of their health problems and their reasons for attending $A \& E$ shortly after arriving in the department, to be compared directly with the actual diagnoses and processes of care received. The shortfall in recruiting 267 to complete the questionnaire rather than the target of 294 occurred in the initial stages of the study. This was attributable to misunderstandings between staff about the processes entailed rather than patients refusing to participate and was resolved by improved communication and monitoring. Potentially, selection bias could have occurred in the recruiting but we were able to confirm by cross checking the numbers assigned to the questionnaires at the point of recruitment against the sequence of numbers printed electronically on the $A \& E$ notes at the time of registration, that bias from this source was minimal. 
The process of grading the strengths of the reasons given by patients for attending $A \& E$ was subjective. However, each member of the research team graded the reasons independently, and differences in scores were resolved in a panel discussion. We acknowledge the complexities in patients' consulting behaviour reported elsewhere, ${ }^{21}$ and recognise that interaction between different reasons is unlikely to be constant. However, the sensitivity tests we conducted around the classification of the reasons made only marginal differences to some of the results, and had no effect on the key finding, which is in accord with the results of recent evaluations of new services, ${ }^{1122}$ that observed no effect on attendances at $\mathrm{A} \& \mathrm{E}$ departments.

FUTURE RESEARCH

A proliferation of services other than $A \& E$ where patients with non-urgent health problems might present, seems to widen patient choice and also to offer an opportunity to reduce demands on $\mathrm{A} \& \mathrm{E}$. However, looking at the patterns of reasons for attending A\&E provided by the patient, we found a strong impetus to continue to present to $\mathrm{A} \& \mathrm{E}$. The challenge in the context of managing non-urgent $\mathrm{A} \& \mathrm{E}$ caseload therefore is to focus less on the clinical needs of patients and on developing services remote from $A \& E$ to meet these needs, in favour of improving our understanding of patient consulting behaviour to facilitate the appropriate matching of service to patient perceptions of need.

The authors would like to thank Mr J Wardrope, consultant in $A \& E$, all the nursing and medical staff at the Northern General Hospital who helped with this study, and Julie Perrin and her nurse practitioner colleagues for advice on the application of the protocols used in the minor injury unit at the Royal Hallamshire Hospital, Sheffield.

Contributors

This study formed a separate and discrete part of a larger project. Jon Nicholl was the lead applicant and prepared the funding application. Jon Nicholl, Pat Coleman, and Richard Irons discussed core themes and ideas for this study. Richard Irons contributed knowledge of the patient care processes and information systems in the A\&E department Richard Irons information systems in the A\&E department. Richard Irons adapted the ideas for implementing in a service setting. Pat Coleman designed the questionnaire and all the other documentation. Pat Coleman and Richard Irons collaborated in developing the processes of data collection. Pat Coleman obtained approval for the methods from the local ethics committee. Richard Irons and Pat Coleman piloted and communicated with the nursing and medical staff and preserved communicated with the nursing and medical staff and preserved the integrity of the methods around recruiting of patients and collecting data at different times in a 24 hour period, continuously for the duration of the study. Pat Coleman processed the data and carried out the preliminary analysis. Jon Nicholl, Pat Coleman and Richard Irons discussed the core findings and participated in the further analysis and interpretation. All three authors contributed substantially to the format and content of the paper. Pat Coleman prepared the first draft.
Jon Nicholl edited the final version. Pat Coleman and Jon Nicholl will act as guarantors for the paper.

Funding: the study was funded by the NHS Executive Trent, but the views expressed in the paper are those of the authors alone and do not necessarily represent the views of the NHS Executive Trent.

Conflicts of interest: none.

1 By accident or design. London: HMSO, 1996.

2 Murphy AW, Leonard C, Plunkett PK, et al. Effect of the introduction of a financial incentive for fee-paying $A \& E$ attenders to consult their GP before attending the A\&E department. Fam Pract 1997;14:407-10.

3 Dale J, Green J, Reid F, et al. Primary care in the accident and emergency department; II. comparison of general and emergency department; II. comparison of general

4 Murphy AW, Bury G, Plunket PK, et al. Randomised controlled trial of general practitioner versus usual medical care in an urban accident and emergency department: process, outcome and comparative cost. BMF 1996;312: $1135-42$.

5 Dale J, Laing H, Roberts AJ, et al. Cost effectiveness of treating primary care patients in accident and emergency; a comparison between GP, senior house officers and registrars. BMF 1996;312:1340-4

6 Ward P, Huddy J, Hargreaves S, et al. Primary care in London: an evaluation of GPs working in an inner city accident and emergency department. F Accid Emerg Med 1996;13:11-15.

7 Gibney D, AW Murphy, Barton D, et al. Randomised controlled trial of general practitioner versus usual medical
care in a suburban accident and emergency department using an informal triage system. Br 7 Gen Pract 1999;49: using

8 Mabrook AF, Dale B. Can nurse practitioners offer a qualty service? An evaluation of a year's work of a nurse led minor injury unit. $\mathcal{F}$ Accid Emerg Med 1998;15:255-68.

9 Sakr M, Angus J, Perrin J, et al. Care of minor injuries by emergency nurse practitioners or junior doctors: a randomised controlled trial. Lancet 1999;354:1321-6.

10 Department of Health. NHS primary care walk-in-centres selection of pilot sites for 1999/2000. Health Services Circular 1999/116. London: Department of Health, 1999.

11 Munro J, Nicholl J, O'Cathain A, et al. Impact of NHS Direct on demand for immediate care: observational study. BMF 2000;321:150-3.

12 Young GP, Wagner MB, Kellermann AL, et al. Ambulatory visits to hospital emergency departments. $7 A M A$ 1996;276: $460-5$.

13 Davison AG, Hindrey ACC, Floyer MA. Use and misuse of an accident and emergency department in the East End of London. $7 R$ Soc Med 1983;76:37-40.

14 Green J, Dale J. Primary care in accident and emergency and general practice: a comparison. Soc Sci Med 1992;35: and general

15 International Classification of Diseases 9th Revision Clinical Modification Third Edition. Volume 1. DHHS Publication no. (PHS) 89-1260. US Department of Health and Human Services, March 1989.

16 Lowy A, Kohler B, Nicholl J. Attendance at accident and emergency departments; unnecessary or inappropriate? $\mathcal{f}$ Public Health Med 1994;16:134-40.

17 Robertson-Steel JRS. Providing primary care in the accident and emergency department. The end of the inappropriate attender. BMF 1998;316:409-10.

18 Patients with minor injuries: a literature review of options for their treatment outside major accident and emergency departments or occupational health settings. Trent Institute for Health Services Research discussion paper no 1, September 1994.

19 Department of Health. The new NHS: modern and dependable. London: Stationery Office, 1997.

20 Midgley S. Choosing the right remedy. NHS Magazine 1999; 19 Winter: 8-9.

21 Rogers A, Hassell K, Nicolaas G. Demanding patients? Analysing the use of primary care. Buckingham: Open University Press, 1998

22 Lattimer V, George S, Thompson F, et al. Safety and effectiveness of nurse telephone conultation in out of hours primary care; randomised controlled trial. The South Wilshire Out of Hours Project (SWOOP) Group. BMF 1998;317: $1054-9$ 